\title{
Numerical Oscillations Analysis for Nonlinear Delay Differential Equations in Physiological Control Systems
}

\section{Qi Wang and Jiechang Wen}

School of Applied Mathematics, Guangdong University of Technology, Guangzhou 510006, China

Correspondence should be addressed to Qi Wang, bmwzwq@126.com

Received 29 August 2012; Accepted 8 December 2012

Academic Editor: Mehmet Sezer

Copyright (C) 2012 Q. Wang and J. Wen. This is an open access article distributed under the Creative Commons Attribution License, which permits unrestricted use, distribution, and reproduction in any medium, provided the original work is properly cited.

This paper deals with the oscillations of numerical solutions for the nonlinear delay differential equations in physiological control systems. The exponential $\theta$-method is applied to $p^{\prime}(t)=$ $\beta_{0} \omega^{\mu} p(t-\tau) /\left(\omega^{\mu}+p^{\mu}(t-\tau)\right)-\gamma p(t)$ and it is shown that the exponential $\theta$-method has the same order of convergence as that of the classical $\theta$-method. Several conditions under which the numerical solutions oscillate are derived. Moreover, it is proven that every nonoscillatory numerical solution tends to positive equilibrium of the continuous system. Finally, the main results are illustrated with numerical examples.

\section{Introduction}

The nonlinear delay differential equation

$$
p^{\prime}(t)=\frac{\beta_{0} \omega^{\mu} p(t-\tau)}{\omega^{\mu}+p^{\mu}(t-\tau)}-\gamma p(t)
$$

where

$$
\omega>0, \quad \beta_{0}>\gamma>0, \quad \mu, \tau \in \mathbf{R}^{+},
$$

has been proposed by Mackey and Glass [1] as model of hematopoiesis (blood cell production). Here, $p(t)$ denotes the density of mature cells in blood circulation, $\tau$ is the time 
delay between the production of immature cells in the bone marrow and their maturation for release in the circulating blood stream, and the production is a single-humped function of $p(t-\tau)$. Equation (1.1) has been recently studied by many authors. Mackey and Heidn [2] considered the local asymptotic stability of the positive equilibrium by the well-known technique of linearization. Gopalsamy et al. [3] obtained sufficient and also necessary conditions for all positive solutions to oscillate about their positive steady states. They also obtained sufficient conditions for the positive equilibrium to be a global attractor. For more details of (1.1), we refer to Mackey [4, 5], and Su et al. [6].

Our aim in this paper is to investigate the oscillations of numerical solutions for (1.1). The oscillatory and asymptotic behavior of solutions of delay differential equations has been the subject of intensive investigations during the past decades. The strong interest in this study is motivated by the fact that it has many useful applications in some mathematical models, such as ecology, biology, and spread of some infectious diseases in humans. The general theory and basic results for this paper have been thoroughly studied in $[7,8]$. In recent years, much research has been focused on the oscillations of numerical solutions for delay differential equations [9-12]. Until now, very few results dealing with the corresponding behavior for nonlinear delay differential equations have been presented in the literature except for [13]. In [13], the authors investigate the oscillations of numerical solutions for the nonlinear delay differential equation of population dynamics. Different from [13], in our paper, we will consider another nonlinear delay differential equation (1.1) in physiological control systems and obtain some new results. We not only investigate some sufficient conditions under which the numerical solutions are oscillatory but also consider the asymptotic behavior of nonoscillatory numerical solutions.

The structure of this paper is as follows. In Section 2, some necessary definitions and results for oscillations of the analytic solutions are given. In Section 3, we obtain the numerical discrete equation by applying the $\theta$-methods to the simplified form which comes from making two transformations on (1.1). Moreover, the oscillations of the numerical solutions are discussed, and conditions under which the numerical solutions oscillate are obtained. In Section 4, we investigate the asymptotic behavior of nonoscillatory solutions. In Section 5, we present numerical examples that illustrate the theoretical results for the numerical methods.

\section{Preliminaries}

Let us state some definitions, lemmas, and theorems that will be used throughout this paper.

Definition 2.1. A function $p$ of (1.1) is said to oscillate about $M^{*}$ if $p-M^{*}$ has arbitrarily large zeros. Otherwise, $p$ is called non-oscillatory. When $M^{*}=0$, we say that $p$ oscillates about zero or simply oscillates.

Definition 2.2. A sequence $\left\{p_{n}\right\}$ is said to oscillate about $\left\{z_{n}\right\}$ if $\left\{p_{n}-z_{n}\right\}$ is neither eventually positive nor eventually negative. Otherwise, $\left\{p_{n}\right\}$ is called non-oscillatory. If $\left\{z_{n}\right\}=\{z\}$ is a constant sequence, we simply say that $\left\{p_{n}\right\}$ oscillates about $\{z\}$. When $\left\{z_{n}\right\}=\{0\}$, we say that $\left\{p_{n}\right\}$ oscillates about zero or simply oscillates.

Definition 2.3. We say that (1.1) oscillates if all of its solutions are oscillatory. 
Theorem 2.4 (see [14]). Consider the difference equation

$$
a_{n+1}-a_{n}+\sum_{j=-k}^{l} q_{j} a_{n+j}=0
$$

and assume that $k, l \in \mathbf{N}$ and $q_{j} \in \mathbf{R}$ for $j=-k, \ldots, l$. Then, the following statements are equivalent:

(1) every solution of (2.1) oscillates;

(2) the characteristic equation $\lambda-1+\sum_{j=-k}^{l} q_{j} \lambda^{j}=0$ has no positive roots.

Theorem 2.5 (see [14]). Consider the difference equation

$$
a_{n+1}-a_{n}+s a_{n-k}+q a_{n}=0,
$$

where $k>0, p>0$, and $q>0$. Then, the necessary and sufficient conditions for the oscillation of all solutions of $(2.2)$ are $q \in(0,1)$ and

$$
s \frac{(k+1)^{k+1}}{k^{k}}>(1-q)^{k+1} .
$$

Lemma 2.6. The inequality $\ln (1+x)>x /(1+x)$ holds for $x>-1$ and $x \neq 0$.

Lemma 2.7. The inequality $e^{x}<1 /(1-x)$ holds for $x<-1$ and $x \neq 0$.

Lemma 2.8 (see [15]). For all $m \geq M_{0}$, one has

(1) $(1+a /(m-\theta a))^{m} \geq e^{a}$ if and only if $1 / 2 \leq \theta \leq 1$ for $a>0, \varphi(-1) \leq \theta \leq 1$ for $a<0$;

(2) $(1+a /(m-\theta a))^{m}<e^{a}$ if and only if $0 \leq \theta<1 / 2$ for $a<0,0 \leq \theta \leq \varphi$ (1) for $a>0$,

where $\varphi(x)=1 / x-1 /\left(e^{x}-1\right)$ and $M_{0}$ is a positive constant.

\section{Oscillations of Numerical Solutions}

\subsection{Two Transformations}

In order to study (1.1) conveniently, we will impose two transformations on (1.1) in this subsection.

Together with (1.1), we will consider the initial condition,

$$
p(t)=\psi(t), \quad-\tau \leq t \leq 0,
$$

the initial value problem (1.1), and (3.1) has a unique positive solution for all $t \geq 0$.

We introduce a similar method in [3]. The change of variables

$$
p(t)=\omega x(t)
$$


turns (1.1) into the delay differential equation

$$
x^{\prime}(t)=\beta_{0} \frac{x(t-\tau)}{1+x^{\mu}(t-\tau)}-\gamma x(t)
$$

with positive equilibrium $M$, which is denoted as

$$
M=\left(\frac{\beta_{0}-\gamma}{\gamma}\right)^{1 / \mu}
$$

The following theorem gives oscillations of the analytic solution of (3.3).

Theorem 3.1 (see [3]). Assume that

$$
\begin{array}{r}
\mu>1, \quad \frac{\beta_{0}}{\gamma}>\frac{\mu}{\mu-1}, \\
e^{\gamma \tau} \frac{\gamma}{\beta_{0}}\left((\mu-1) \beta_{0}-\mu \gamma\right) \tau>\frac{1}{e},
\end{array}
$$

then every positive solution of (3.3) oscillates about its positive equilibrium M.

The following corollary is naturally obtained.

Corollary 3.2. Assume that all the conditions in Theorem 3.1 hold, then every positive solution of (1.1) oscillates about its positive equilibrium $M^{*}=\omega M$.

Next, we introduce an invariant oscillation transformation $x(t)=M e^{y(t)}$, and then (3.3) can be written as

$$
y^{\prime}(t)+\frac{\beta_{0}}{1+M^{\mu}}\left[f_{1}(y(t)) f_{2}(y(t-\tau))-f_{1}(y(t))-f_{2}(y(t-\tau))+2\right]=0,
$$

where

$$
f_{1}(u)=1-e^{-u}, \quad f_{2}(u)=1+\frac{\left(1+M^{\mu}\right) e^{u}}{1+M^{\mu} e^{\mu u}} .
$$

Then, $x(t)$ oscillates about $M$ if and only if $y(t)$ oscillates about zero.

Moreover, since

$$
\frac{\beta_{0}}{1+M^{\mu}}=\gamma,
$$


then (3.6) and (3.7) become

$$
\begin{gathered}
e^{\gamma \tau} \gamma\left(\frac{\mu M^{\mu}}{1+M^{\mu}}-1\right) \tau>\frac{1}{e}, \\
y^{\prime}(t)=-\gamma f_{1}(y(t)) f_{2}(y(t-\tau))+\gamma f_{1}(y(t))+\gamma f_{2}(y(t-\tau))-2 \gamma,
\end{gathered}
$$

respectively.

For our convenience, denote

$$
Q=\frac{\mu M^{\mu}}{1+M^{\mu}}-1
$$

then the inequality (3.10) yields

$$
e^{\gamma \tau} Q \gamma \tau>\frac{1}{e}
$$

\subsection{The Difference Scheme}

Let $h=\tau / m$ be a given stepsize with integer $m>1$. The adaptation of the linear $\theta$-method and the one-leg $\theta$-method to (3.11) leads to the same numerical process of the following type:

$$
\begin{aligned}
y_{n+1}= & y_{n}-h \theta \gamma f_{1}\left(y_{n+1}\right) f_{2}\left(y_{n+1-m}\right)-h(1-\theta) \gamma f_{1}\left(y_{n}\right) f_{2}\left(y_{n-m}\right)+h \theta \gamma f_{1}\left(y_{n+1}\right) \\
& +h(1-\theta) \gamma f_{1}\left(y_{n}\right)+h \theta \gamma f_{2}\left(y_{n+1-m}\right)+h(1-\theta) \gamma f_{2}\left(y_{n-m}\right)-2 h \gamma,
\end{aligned}
$$

where $0 \leq \theta \leq 1, y_{n+1}$ and $y_{n+1-m}$ are approximations to $y(t)$ and $y(t-\tau)$ of (3.11) at $t_{n+1}$, respectively.

Letting $y_{n}=\ln \left(p_{n} / M^{*}\right)$ and using the expressions of $f_{1}$ and $f_{2}$, we have

$$
p_{n+1}=p_{n} \exp \left(h \gamma \omega^{\mu}\left(1+M^{\mu}\right)\left(\frac{\theta p_{n+1-m}}{p_{n+1}\left(\omega^{\mu}+p_{n+1-m}^{\mu}\right)}+\frac{(1-\theta) p_{n-m}}{p_{n}\left(\omega^{\mu}+p_{n-m}^{\mu}\right)}\right)-h \gamma\right) .
$$

Definition 3.3. We call the iteration formula (3.15) the exponential $\theta$-method for (1.1), where $p_{n+1}$ and $p_{n+1-m}$ are approximations to $p(t)$ and $p(t-\tau)$ of $(1.1)$ at $t_{n+1}$, respectively.

The following theorem, for the proof of which we refer to [16], allows us to obtain the convergence of exponential $\theta$-method.

Theorem 3.4. The exponential $\theta$-method (3.15) is convergent with order

$$
\begin{aligned}
& \text { 1, when } \theta \neq \frac{1}{2} \\
& \text { 2, when } \theta=\frac{1}{2} \text {. }
\end{aligned}
$$




\subsection{Oscillation Analysis}

It is not difficult to know that $p_{n}$ oscillates about $M^{*}$ if and only if $y_{n}$ is oscillatory. In order to study oscillations of (3.15), we only need to consider the oscillations of (3.14). The following conditions which are taken from [3] will be used in the next analysis:

$$
\begin{gathered}
u f_{1}(u)>0, \quad \text { for } u \neq 0, \quad \lim _{u \rightarrow 0} \frac{f_{1}(u)}{u}=1, \\
f_{2}(u)>0, \quad \text { for every } u, \quad \lim _{u \rightarrow 0} f_{2}(u)=2, \\
f_{1}(u) \leq u, \text { for } u>0, \quad f_{1}(0)=0, \\
f_{2}(u) \leq 2, \quad \text { for } u \geq 0, \quad \mu>2, M^{\mu}>1, \quad f_{2}(0)=2 .
\end{gathered}
$$

The linearized form of (3.14) is given by

$$
\begin{aligned}
y_{n+1}= & y_{n}-h \theta \gamma y_{n+1}-h(1-\theta) \gamma y_{n}+h \theta \gamma\left(1-\frac{\mu M^{\mu}}{1+M^{\mu}}\right) y_{n+1-m} \\
& +h(1-\theta) \gamma\left(1-\frac{\mu M^{\mu}}{1+M^{\mu}}\right) y_{n-m} .
\end{aligned}
$$

Then by (3.12), (3.18) gives

$$
y_{n+1}=\frac{1-h(1-\theta) \gamma}{1+h \theta \gamma} y_{n}-\frac{h \theta \gamma Q}{1+h \theta \gamma} y_{n+1-m}-\frac{h(1-\theta) \gamma Q}{1+h \theta \gamma} y_{n-m}
$$

It follows from [14] that (3.14) oscillates if (3.19) oscillates under the condition (3.17).

Definition 3.5. Equation (3.15) is said to be oscillatory if all of its solutions are oscillatory.

Definition 3.6. We say that the exponential $\theta$-method preserves the oscillations of (1.1) if (1.1) oscillates, then there is a $\bar{h}>0$ or $\bar{h}=\infty$, such that (3.15) oscillates for $h<\bar{h}$. Similarly, we say that the exponential $\theta$-method preserves the nonoscillations of (1.1) if (1.1) non-oscillates, then there is a $\bar{h}>0$ or $\bar{h}=\infty$, such that (3.15) nonoscillates for $h<\bar{h}$.

In the following, we will study whether the exponential $\theta$-method inherits the oscillations of (1.1). Equivalently, when Corollary 3.2 holds, we will investigate the conditions under which (3.15) is oscillatory.

Lemma 3.7. The characteristic equation of (3.18) is given by

$$
\xi=R\left(-h \gamma\left(1+Q \xi^{-m}\right)\right)
$$


Proof. Letting $y_{n}=\xi^{n} y_{0}$ in (3.18), we have

$$
\begin{aligned}
\xi^{n+1} y_{0}= & \xi^{n} y_{0}-h \theta \gamma \xi^{n+1} y_{0}-h(1-\theta) \gamma \xi^{n} y_{0}+h \theta \gamma\left(1-\frac{\mu M^{\mu}}{1+M^{\mu}}\right) \xi^{n+1-m} y_{0} \\
& +h(1-\theta) \gamma\left(1-\frac{\mu M^{\mu}}{1+M^{\mu}}\right) \xi^{n-m} y_{0}
\end{aligned}
$$

that is,

$$
\xi=1-h \theta \gamma \xi\left(1-\left(1-\frac{\mu M^{\mu}}{1+M^{\mu}}\right) \xi^{-m}\right)-h(1-\theta) \gamma\left(1-\left(1-\frac{\mu M^{\mu}}{1+M^{\mu}}\right) \xi^{-m}\right)
$$

which is equivalent to

$$
\begin{aligned}
\xi & =\frac{1-h(1-\theta) \gamma\left(1-\left(1-\left(\mu M^{\mu}\right) /\left(1+M^{\mu}\right)\right) \xi^{-m}\right)}{1+h \theta \gamma\left(1-\left(1-\left(\mu M^{\mu}\right) /\left(1+M^{\mu}\right)\right) \xi^{-m}\right)} \\
& =1-\frac{h \gamma\left(1-\left(1-\left(\mu M^{\mu}\right) /\left(1+M^{\mu}\right)\right) \xi^{-m}\right)}{1+h \theta \gamma\left(1-\left(1-\left(\mu M^{\mu}\right) /\left(1+M^{\mu}\right)\right) \xi^{-m}\right)} .
\end{aligned}
$$

In view of [17], we know that the stability function of the $\theta$-method is

$$
R(x)=\frac{1+(1-\theta) x}{1-\theta x}=1+\frac{x}{1-\theta x} .
$$

By noticing (3.12), thus the characteristic equation of (3.18) is given by (3.20). The proof is completed.

Lemma 3.8. If condition (3.13) holds, then the characteristic equation (3.20) has no positive roots for $0 \leq \theta \leq 1 / 2$.

Proof. Let $f(\xi)=\xi-R\left(-h \gamma\left(1+Q \xi^{-m}\right)\right)$. By Lemma 2.8, we know that

$$
R\left(-h \gamma\left(1+Q \xi^{-m}\right)\right) \leq \exp \left(-h \gamma\left(1+Q \xi^{-m}\right)\right)
$$

holds for $\xi>0$ and $0 \leq \theta \leq 1 / 2$. In the following, we will prove that $g(\xi)=\xi-\exp (-h \gamma(1+$ $\left.\left.Q \xi^{-m}\right)\right)>0$ for $\xi>0$. Suppose the opposite, that is, there exists a $\xi_{0}>0$ such that $W\left(\xi_{0}\right) \leq 0$, then we get $\xi_{0} \leq \exp \left(-h \gamma\left(1+Q \xi_{0}^{-m}\right)\right)$, and 


$$
\xi_{0}^{m} \leq \exp \left(-\gamma \tau-\gamma \tau Q \xi_{0}^{-m}\right)
$$

Multiplying both sides of the inequality (3.26) by $e^{\gamma \tau} Q \gamma \tau e \xi_{0}^{-m}$, we have

$$
e^{\gamma \tau} Q \gamma \tau e \leq Q \gamma \tau \xi_{0}^{-m} \exp \left(1-Q \gamma \tau \xi_{0}^{-m}\right)
$$

Thus, we have the following two cases.

Case 1. If $1-Q \gamma \tau \xi_{0}^{-m}=0$, then $e^{\gamma \tau} Q \gamma \tau e \leq 1$, which contradicts the condition (3.13).

Case 2. If $1-Q \gamma \tau \xi_{0}^{-m} \neq 0$, then in view of Lemma 2.7, we obtain

$$
\exp \left(1-Q \gamma \tau \xi_{0}^{-m}\right)<\frac{1}{1-\left(1-Q \gamma \tau \xi_{0}^{-m}\right)}=\frac{1}{Q \gamma \tau \xi_{0}^{-m}}
$$

that is,

$$
Q \gamma \tau \xi_{0}^{-m} \exp \left(1-Q \gamma \tau \xi_{0}^{-m}\right)<1
$$

so $e^{\gamma \tau} Q_{\gamma \tau e}<1$, which is also a contradiction to (3.13).

In summary, we have, for $\xi>0$,

$$
f(\xi)=\xi-R\left(-h \gamma\left(1+Q \xi^{-m}\right)\right) \geq \xi-\exp \left(-h \gamma\left(1+Q \xi^{-m}\right)\right)=g(\xi)>0
$$

which implies that the characteristic equation (3.20) has no positive roots. The proof is complete.

Without loss of generality, in the case of $1 / 2<\theta \leq 1$, we assume that $m>1$.

Lemma 3.9. If condition (3.13) holds and $1 / 2<\theta \leq 1$, then the characteristic equation (3.20) has no positive roots for $h<\widehat{h}$, where

$$
\widehat{h}= \begin{cases}\infty, & \text { for } Q \gamma \tau \geq 1 \\ \frac{\tau(1+\gamma \tau+\ln Q \gamma \tau)}{1+\gamma \tau(1-\ln Q \gamma \tau)}, & \text { for } Q \gamma \tau<1\end{cases}
$$

Proof. Since $R\left(-h \gamma\left(1+Q \xi^{-m}\right)\right)$ is an increasing function of $\theta$ when $\xi>0$, then, for $\xi>0$ and $1 / 2<\theta \leq 1$,

$$
R\left(-h \gamma\left(1+Q \xi^{-m}\right)\right)=\frac{1-h(1-\theta) \gamma\left(1+Q \xi^{-m}\right)}{1+h \theta \gamma\left(1+Q \xi^{-m}\right)} \leq \frac{1}{1+h \gamma\left(1+Q \xi^{-m}\right)}
$$


In the following, we will prove that the inequality

$$
\xi-\frac{1}{1+h \gamma\left(1+Q \xi^{-m}\right)}>0
$$

holds under certain conditions.

The left side of inequality (3.33) can be rewritten as

$$
\xi-\frac{1}{1+h \gamma\left(1+Q \xi^{-m}\right)}=\frac{(1+h \gamma) \xi^{1-m}}{1+h \gamma\left(1+Q \xi^{-m}\right)} \Gamma(\xi),
$$

where

$$
\Gamma(\xi)=\xi^{m}-\frac{1}{1+h \gamma} \xi^{m-1}+\frac{h \gamma Q}{1+h \gamma},
$$

so we only need to prove that $\Gamma(\xi)>0$ for $\xi>0$. It is easy to know that $\Gamma(\xi)=0$ is the characteristic equation of the following difference equation

$$
y_{n+1}-y_{n}+\frac{h \gamma Q}{1+h \gamma} y_{n+1-m}+\frac{h \gamma}{1+h \gamma} y_{n}=0
$$

By Theorems 2.4 and 2.5, we know that $\Gamma(\xi)$ has no positive roots if and only if

$$
\frac{h \gamma Q}{1+h \gamma} \frac{m^{m}}{(m-1)^{m-1}}>\left(1-\frac{h \gamma}{1+h \gamma}\right)^{m}
$$

which is equivalent to

$$
\ln Q \gamma \tau+(m-1) \ln \left(1+\frac{1+\gamma \tau}{m-1}\right)>0
$$

We examine two cases depending on the position of $Q_{\gamma \tau}$ : either $Q_{\gamma \tau} \geq 1$ or $Q \gamma \tau<1$.

Case 1. If $Q_{\gamma \tau} \geq 1$, by $m>1$, (3.38) holds true.

Case 2. If $Q \gamma \tau<1$ and

$$
h<\frac{\tau(1+\gamma \tau+\ln Q \gamma \tau)}{1+\gamma \tau(1-\ln Q \gamma \tau)}
$$


then by Lemma 2.6, we have

$$
\begin{aligned}
\ln Q \gamma \tau+(m-1) \ln \left(1+\frac{1+\gamma \tau}{m-1}\right) & >\ln Q \gamma \tau+(m-1) \frac{(1+\gamma \tau) /(m-1)}{1+(1+\gamma \tau) /(m-1)} \\
& =\ln Q \gamma \tau+\frac{(m-1)(1+\gamma \tau)}{m+\gamma \tau}>0 .
\end{aligned}
$$

Therefore, the inequality (3.33) holds for $h<\widehat{h}$, where $\widehat{h}$ is defined in (3.31). So, we get that the following inequality

$$
f(\xi)=\xi-R\left(-h \gamma\left(1+Q \xi^{-m}\right)\right) \geq \xi-\frac{1}{1+h \gamma\left(1+Q \xi^{-m}\right)}>0
$$

holds for $h<\widehat{h}$ and $\xi>0$, which implies that the characteristic equation (3.20) has no positive roots. This completes the proof.

Remark 3.10. By inequality (3.38) and condition $Q_{\gamma \tau}<1$, we have

$$
\frac{\tau(1+\gamma \tau+\ln Q \gamma \tau)}{1+\gamma \tau(1-\ln Q \gamma \tau)}>0
$$

thus $\widehat{h}$ is meaningful.

In view of (3.17), Lemmas 3.8 and 3.9, and Theorem 2.4, we present the first main theorem of this paper.

Theorem 3.11. If condition (3.13) holds, then (3.15) is oscillatory for

$$
h<\tilde{h}=\left\{\begin{array}{l}
\infty, \quad \text { when } 0 \leq \theta \leq \frac{1}{2}, \\
\widehat{h}, \quad \text { when } \frac{1}{2}<\theta \leq 1,
\end{array}\right.
$$

where $\widehat{h}$ is defined in Lemma 3.9.

\section{Asymptotic Behavior of Nonoscillatory Solutions}

In this section, we will study the asymptotic behavior of non-oscillatory solutions of (3.15). We first recall the following result about asymptotic behavior of (3.3).

Lemma 4.1 (see [3]). Assume that

$$
\tau>0, \quad \mu>1, \quad \beta_{0}>\gamma,
$$


then every solution $x(t)$ of the initial value problem is

$$
\begin{gathered}
x^{\prime}(t)=\beta_{0} \frac{x(t-\tau)}{1+x^{\mu}(t-\tau)}-\gamma x(t), \\
x(t)=\varphi, \quad-\tau \leq t \leq 0,
\end{gathered}
$$

which is non-oscillatory about $M$ satisfies

$$
\lim _{t \rightarrow \infty} x(t)=M
$$

From (3.3) and (3.7), we know that the non-oscillatory solution of (3.7) satisfies $\lim _{t \rightarrow \infty} y(t)=0$ if Lemma 4.1 is satisfied. Furthermore, $\lim _{t \rightarrow \infty} p(t)=M^{*}$ is also obtained. In the following, we will prove that the numerical solution of (1.1) can inherit this property.

Lemma 4.2. Let $y_{n}$ be a non-oscillatory solution of (3.14), then $\lim _{n \rightarrow \infty} y_{n}=0$.

Proof. Without loss of generality, we assume that $y_{n}>0$ for sufficiently large $n$. Then by condition (3.17), we have

$$
f_{1}\left(y_{i}\right)>0, \quad f_{2}\left(y_{i}\right)-1>0, \quad f_{2}\left(y_{i}\right)-2<0,
$$

for sufficiently large $i$. Moreover, it is can be seen from (3.14) that

$$
\begin{aligned}
y_{n+1}-y_{n}= & -h \theta \gamma f_{1}\left(y_{n+1}\right)\left[f_{2}\left(y_{n+1-m}\right)-1\right]-h(1-\theta) \gamma f_{1}\left(y_{n}\right)\left[f_{2}\left(y_{n-m}\right)-1\right] \\
& +h \theta \gamma\left[f_{2}\left(y_{n+1-m}\right)-2\right]+h(1-\theta) \gamma\left[f_{2}\left(y_{n-m}\right)-2\right],
\end{aligned}
$$

which gives

$$
y_{n+1}-y_{n}-h \theta \gamma\left[f_{2}\left(y_{n+1-m}\right)-2\right]-h(1-\theta) \gamma\left[f_{2}\left(y_{n-m}\right)-2\right]<0,
$$

thus we have

$$
y_{n+1}-y_{n}<h \theta \gamma\left[f_{2}\left(y_{n+1-m}\right)-2\right]+h(1-\theta) \gamma\left[f_{2}\left(y_{n-m}\right)-2\right]<0,
$$

then the sequence $\left\{y_{n}\right\}$ is decreasing, and, therefore,

$$
\lim _{n \rightarrow \infty} y_{n}=\eta \in[0, \infty)
$$

Next, we prove that $\eta=0$. If $\eta>0$, then there exist $N \in \mathbf{N}$ and $\varepsilon>0$ such that $0<\eta-\varepsilon<$ $y_{n}<\eta+\varepsilon$ for $n-m>N$. Thus, $y_{n-m}>\eta-\varepsilon$ and $y_{n-m+1}>\eta-\varepsilon$. So, inequality (4.6) yields

$$
y_{n+1}-y_{n}-h \theta \gamma\left[f_{2}(\eta-\varepsilon)-2\right]-h(1-\theta) \gamma\left[f_{2}(\eta-\varepsilon)-2\right]<0,
$$




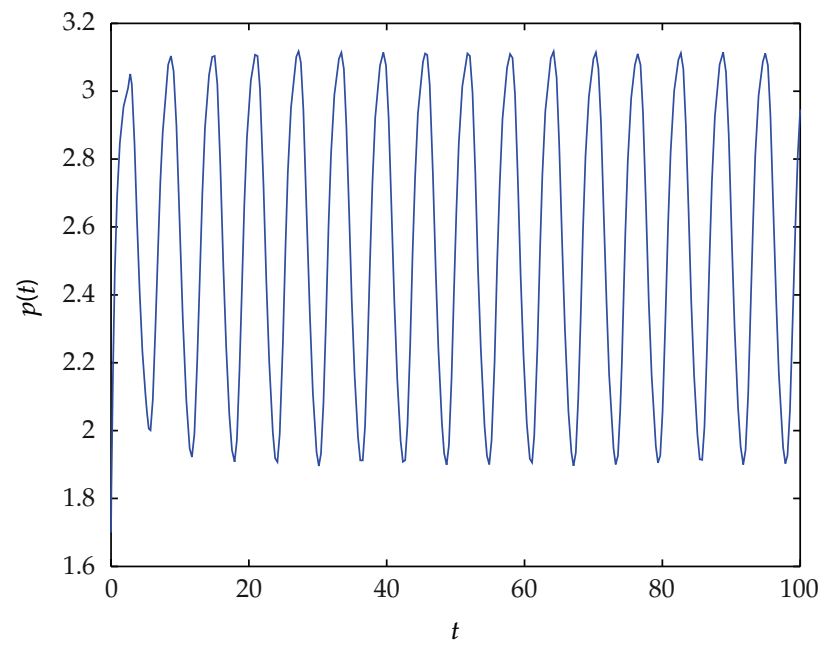

Figure 1: The analytic solution of (5.1).

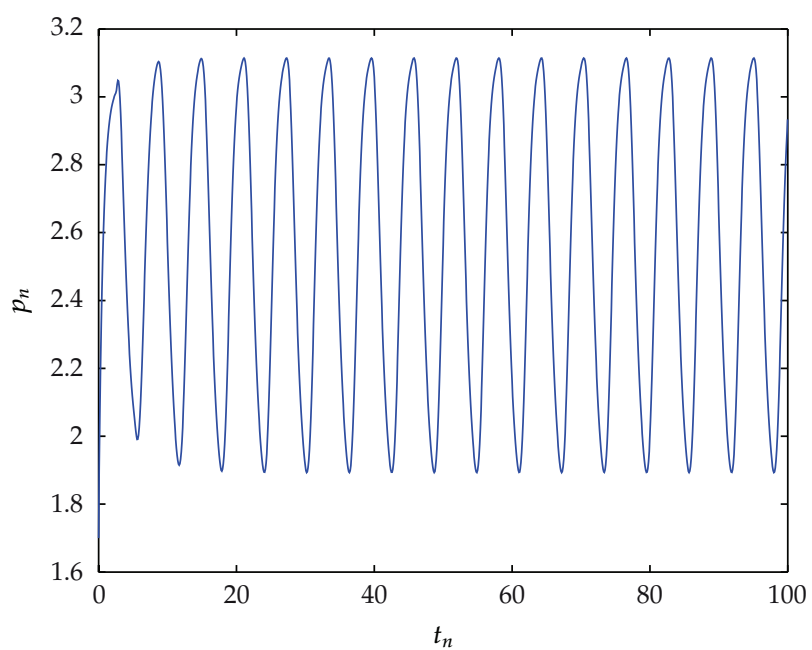

Figure 2: The numerical solution of (5.1) with $m=25$ and $\theta=0.25$.

which implies that $y_{n+1}-y_{n}<C<0$, where

$$
C=h \gamma\left[f_{2}(\eta-\varepsilon)-2\right]=h \gamma \frac{\left(e^{\eta-\varepsilon}-1\right)+M^{\mu} e^{\eta-\varepsilon}\left(1-e^{(\mu-1)(\eta-\varepsilon)}\right)}{1+M^{\mu} e^{\mu(\eta-\varepsilon)}}
$$

Thus, $y_{n} \rightarrow-\infty$ as $n \rightarrow \infty$, which is a contradiction to (4.8). This completes the proof.

As a consequence, the second main theorem of this paper is as follows.

Theorem 4.3. Letting $p_{n}$ be a positive solution of (3.15), which does not oscillate about $M^{*}$, then $\lim _{n \rightarrow \infty} p_{n}=M^{*}$, where $M^{*}$ is the positive equilibrium of the continuous system (1.1). 


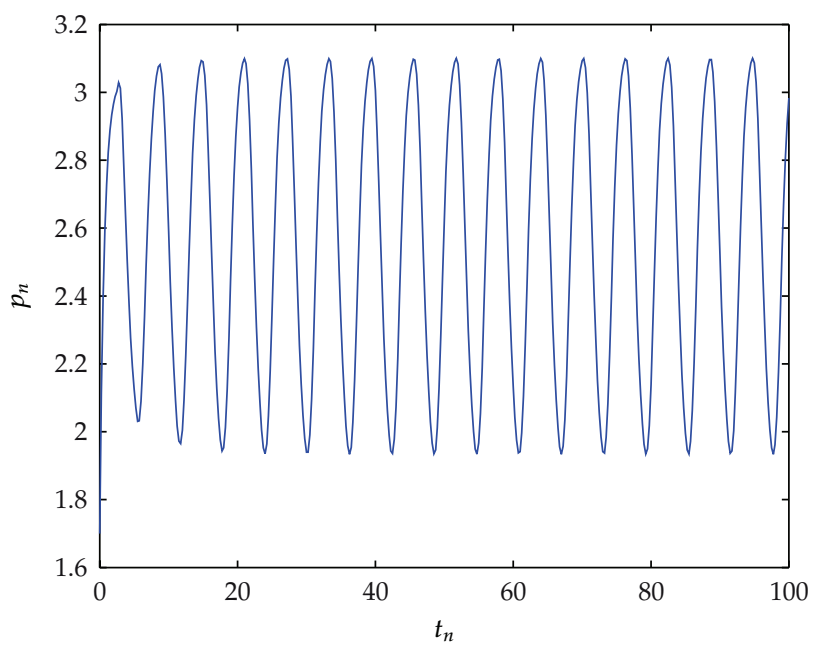

Figure 3: The numerical solution of (5.1) with $m=10$ and $\theta=0.6$.

\section{Numerical Examples}

In order to verify our results, three numerical examples are examined in this section.

Example 5.1. Consider the following equation:

$$
p^{\prime}(t)=\frac{3 \times 2.6^{5} p(t-2.5)}{2.6^{5}+p^{5}(t-2.5)}-1.5 p(t)
$$

subject to the initial condition

$$
p(t)=1.7, \quad \text { for } t \leq 0 \text {. }
$$

In (5.1), it can be seen that condition (3.6) holds true and $Q \gamma \tau \approx 5.6250>1$. That is, the analytic solutions of (5.1) are oscillatory. In Figures 1-3, we draw the figures of the analytic solutions and the numerical solutions of (5.1), respectively. Set $m=25, \theta=0.25$ in Figure 2 and $m=10, \theta=0.6$ in Figure 3, respectively. From the two figures, we can see that the numerical solutions of (5.1) oscillate about $M^{*} \approx 2.6$, which are in agreement with Theorem 3.11.

Example 5.2. Let us consider the equation

$$
p^{\prime}(t)=\frac{1.77 \times 1.98^{3} p(t-2)}{1.98^{3}+p^{3}(t-2)}-p(t),
$$

with the initial value $p(t)=1.9$ for $t \leq 0$. In (5.3), it is easy to see that condition (3.6) is fulfilled and $Q \gamma \tau \approx 0.6102<1$. That is, the analytic solutions of (5.3) are oscillatory. In Figures 4-6, we draw the figures of the analytic solutions and the numerical solutions of (5.3), respectively. 


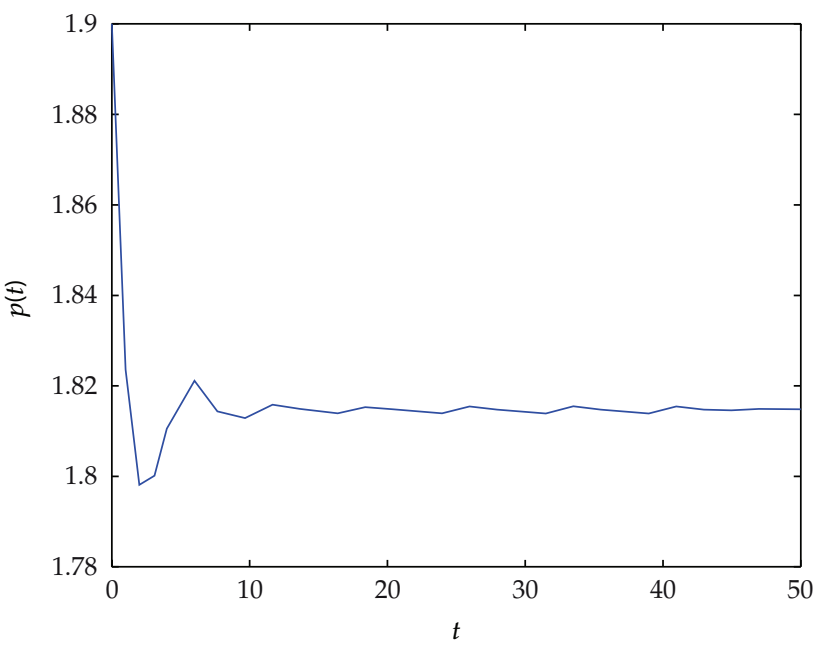

Figure 4: The analytic solution of (5.3).

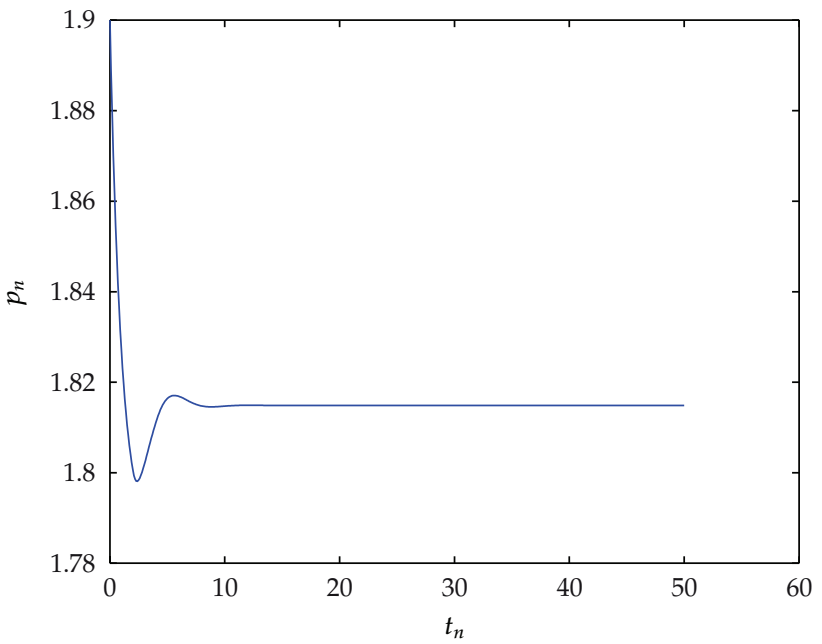

Figure 5: The numerical solution of (5.3) with $m=20$ and $\theta=0.4$.

Set $m=20, \theta=0.4$ in Figure 5 and $m=40, \theta=0.7$ in Figure 6, respectively. We can see from the three figures that the numerical solutions of (5.3) oscillate about $M^{*} \approx 1.8149$, which are consistent with Theorem 3.11. On the other hand, by direct calculation, we get $\widehat{h} \approx 1.2568$, so the stepsize $\widehat{h}$ is not optimal.

Example 5.3. Consider the following equation:

$$
p^{\prime}(t)=\frac{1.5 \times 3.7^{2} p(t-1)}{3.7^{2}+p^{2}(t-1)}-0.5 p(t)
$$




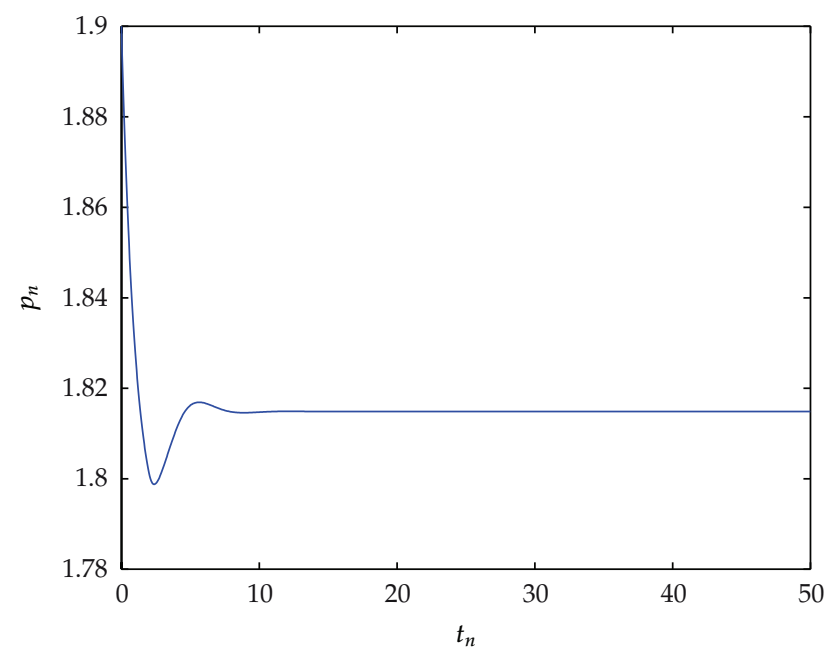

Figure 6: The numerical solution of (5.3) with $m=40$ and $\theta=0.7$.

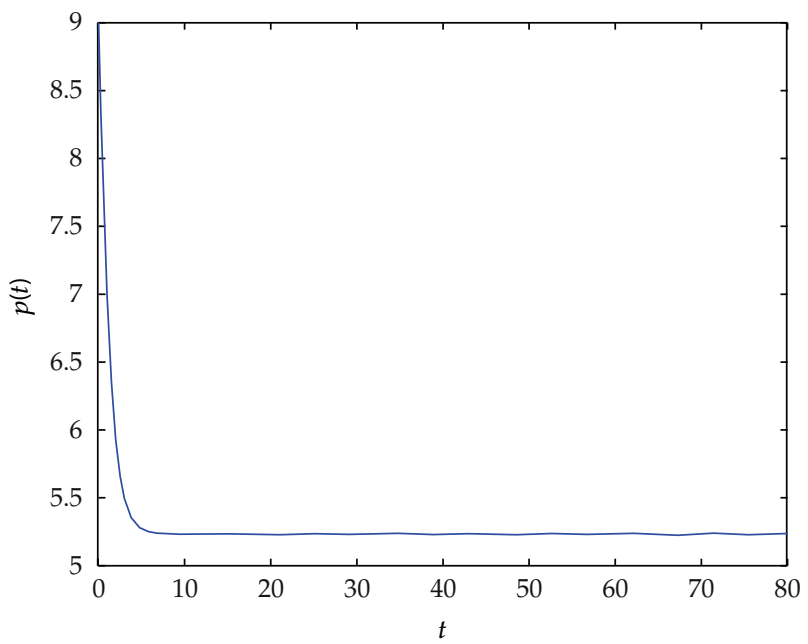

Figure 7: The analytic solution of (5.4).

subject to the initial condition

$$
p(t)=9, \quad \text { for } t \leq 0
$$

For (5.4), it is easy to see that $Q_{\gamma \tau e^{\gamma \tau+1}} \approx 0.7469<1$, so the condition (3.6) is not satisfied. That is, the analytic solutions of (5.4) are non-oscillatory. In Figures 7-9, we draw the figures of the analytic solutions and the numerical solutions of (5.4), respectively. In Figure 7, we can see that $p(t) \rightarrow M^{*} \approx 5.2326$ as $t \rightarrow \infty$. From Figures 8 and 9 , we can also see that the numerical solutions of (5.4) satisfy $p_{n} \rightarrow M^{*} \approx 5.2326$ as $n \rightarrow \infty$. That is, the numerical method inherits the asymptotic behavior of non-oscillatory solutions of (5.4), which coincides with Theorem 4.3. 


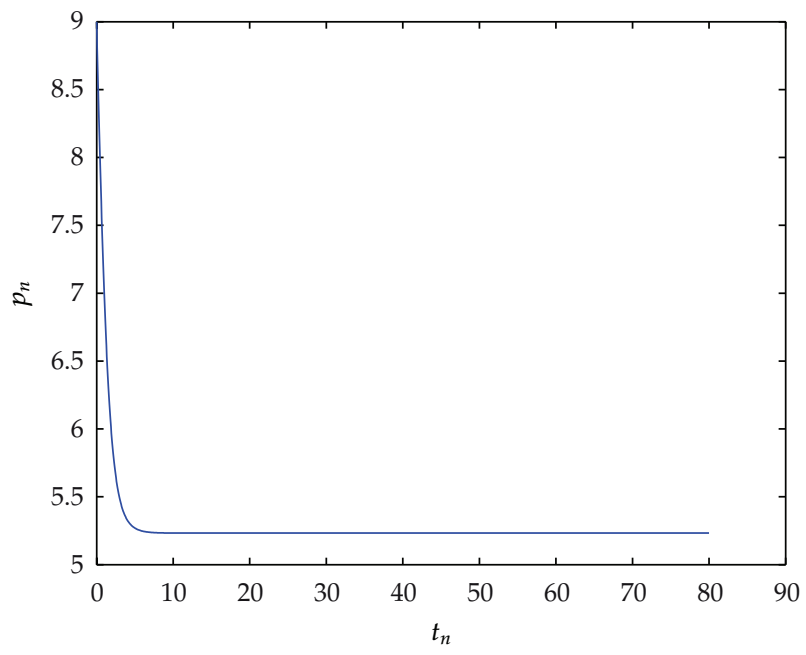

Figure 8: The numerical solution of (5.4) with $m=15$ and $\theta=0.3$.

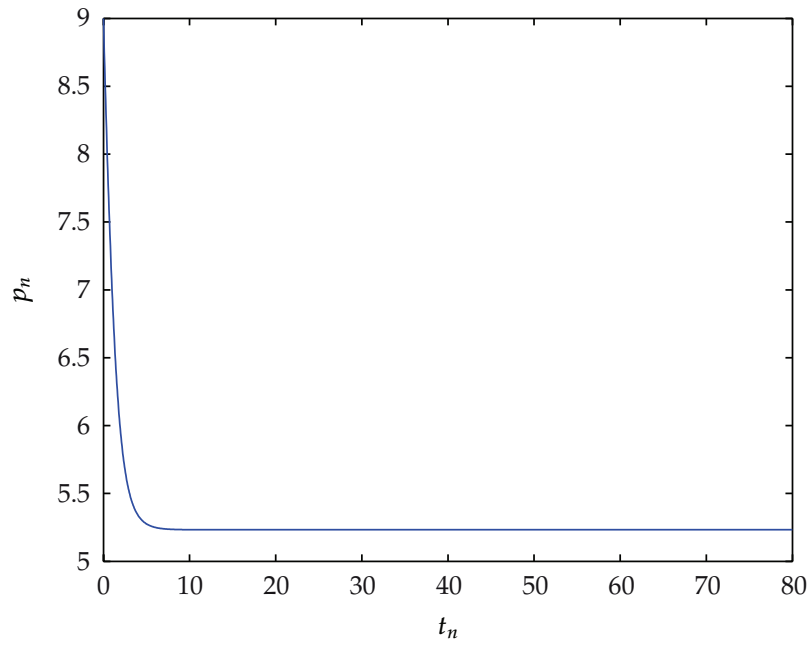

Figure 9: The numerical solution of (5.4) with $m=20$ and $\theta=0.8$.

Furthermore, according to Definition 3.6, we can see from these figures that the exponential $\theta$-method preserves the oscillations of (5.1) and (5.3) and the non-oscillations of (5.4), respectively.

\section{Acknowledgments}

Q. Wang's work was supported by the National Natural Science Foundation of China (no. 11201084). The authors would like to thank Professor Mingzhu Liu, Minghui Song, and Dr. Zhanwen Yang for their useful suggestions. 


\section{References}

[1] M. C. Mackey and L. Glass, “Oscillation and chaos in physiological control systems," Science, vol. 197, no. 4300, pp. 287-289, 1977.

[2] M. C. Mackey and A. D. Heiden, "Dynamical diseases and bifurcations: understanding functional disorders in physiological systems, Funkt," Biology and Medicine, vol. 1, pp. 156-164, 1982.

[3] K. Gopalsamy, M. R. S. Kulenović, and G. Ladas, "Oscillations and global attractivity in models of hematopoiesis," Journal of Dynamics and Differential Equations, vol. 2, no. 2, pp. 117-132, 1990.

[4] M. C. Mackey, "Periodic auto-immune hemolytic anemia: an induced dynamical disease," Bulletin of Mathematical Biology, vol. 41, no. 6, pp. 829-834, 1979.

[5] M. C. Mackey, "Some models in hemopoiesis: predictions and problems," in Biomathematics and Cell Kinetics, Elsevier, Amsterdam, The Netherlands, 1981.

[6] H. Su, X. Ding, and W. Li, "Numerical bifurcation control of Mackey-Glass system," Applied Mathematical Modelling, vol. 35, no. 7, pp. 3460-3472, 2011.

[7] K. Gopalsamy, Stability and Oscillations in Delay Differential Equations of Population Dynamics, vol. 74, Kluwer Academic, Dordrecht, The Netherlands, 1992.

[8] D. D. Baĭnov and D. P. Mishev, Oscillation Theory for Neutral Differential Equations with Delay, Adam Hilger, New York, NY, USA, 1991.

[9] M. Z. Liu, J. Gao, and Z. W. Yang, "Oscillation analysis of numerical solution in the $\theta$-methods for equation $x^{\prime}(t)+a x(t)+a_{1} x([t-1])=0$, , Applied Mathematics and Computation, vol. 186, no. 1, pp. 566-578, 2007.

[10] M. Z. Liu, J. F. Gao, and Z. W. Yang, "Preservation of oscillations of the Runge-Kutta method for equation $x^{\prime}(t)+a x(t)+a_{1} x([t-1])=0$," Computers $\mathcal{E}$ Mathematics with Applications, vol. 58, no. 6, pp. 1113-1125, 2009.

[11] Q. Wang, Q. Zhu, and M. Liu, "Stability and oscillations of numerical solutions for differential equations with piecewise continuous arguments of alternately advanced and retarded type," Journal of Computational and Applied Mathematics, vol. 235, no. 5, pp. 1542-1552, 2011.

[12] J. F. Gao, "Oscillations analysis of numerical solutions for neutral delay differential equations," International Journal of Computer Mathematics, vol. 88, no. 12, pp. 2648-2665, 2011.

[13] J. Gao, M. Song, and M. Liu, "Oscillation analysis of numerical solutions for nonlinear delay differential equations of population dynamics," Mathematical Modelling and Analysis, vol. 16, no. 3, pp. 365-375, 2011.

[14] I. Györi and G. Ladas, Oscillation Theory of Delay Differential Equations With Applications, Academic Press, Oxford, UK, 1991.

[15] M. H. Song, Z. W. Yang, and M. Z. Liu, "Stability of $\theta$-methods for advanced differential equations with piecewise continuous arguments," Computers \& Mathematics with Applications, vol. 49, no. 9-10, pp. 1295-1301, 2005.

[16] J. Hale, Theory of Functional Differential Equations, Springer, New York, NY, USA, 1977.

[17] Z. Yang, M. Liu, and M. Song, "Stability of Runge-Kutta methods in the numerical solution of equation $u^{\prime}(t)=a u(t)+a_{0} u([t])+a_{1} u([t-1])$," Applied Mathematics and Computation, vol. 162, no. 1, pp. 37-50, 2005. 


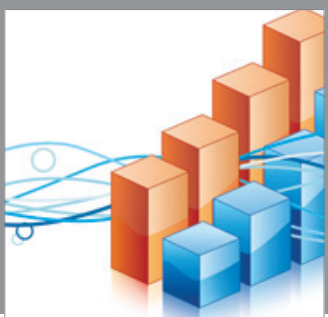

Advances in

Operations Research

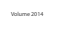

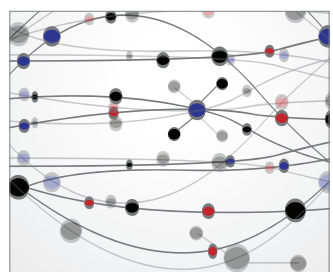

\section{The Scientific} World Journal
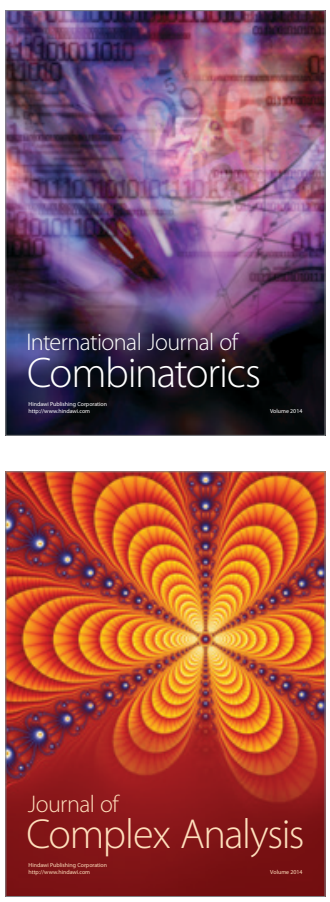

International Journal of

Mathematics and

Mathematical

Sciences
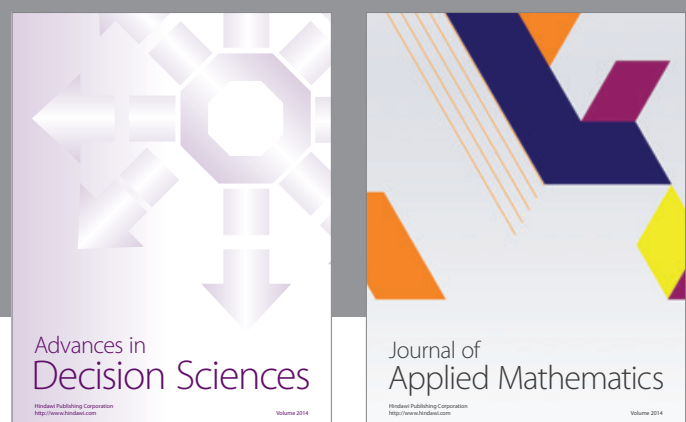

Journal of

Applied Mathematics
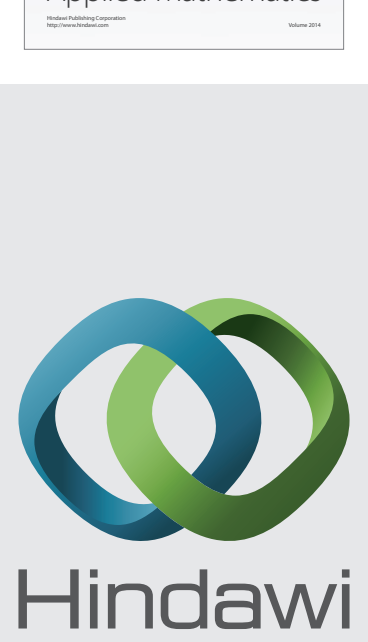

Submit your manuscripts at http://www.hindawi.com
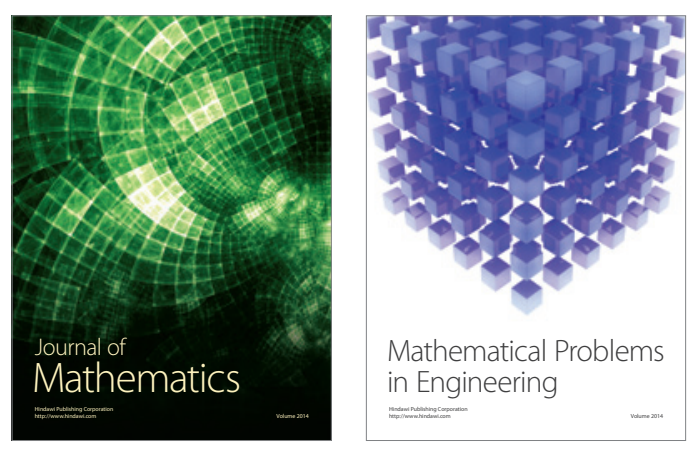

Mathematical Problems in Engineering
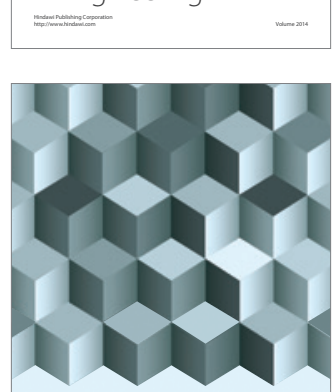

Journal of

Function Spaces
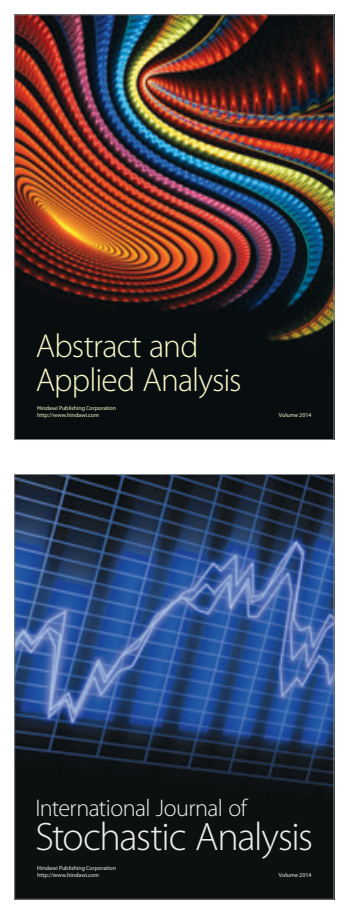

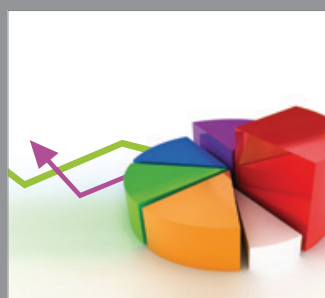

ournal of

Probability and Statistics

Promensencen
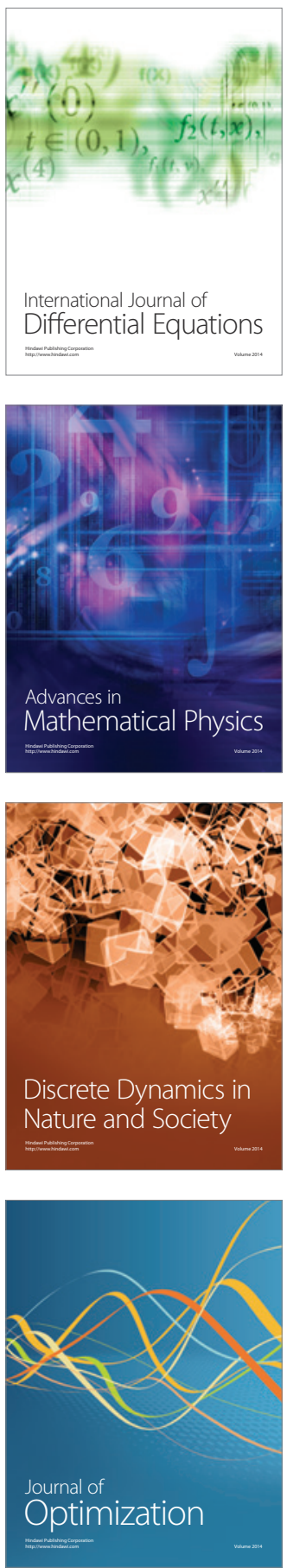